\title{
Specific KRAS amino acid substitutions and EGFR mutations predict site-specific recurrence and metastasis following non-small-cell lung cancer surgery
}

\author{
Stéphane Renaud ${ }^{\star}, 1,2$, Joseph Seitlinger ${ }^{1}$, Pierre-Emmanuel Falcoz ${ }^{1}$, Mickaël Schaeffer ${ }^{3}$, Anne-Claire Voegeli ${ }^{4}$, \\ Michèle Legrain ${ }^{4}$, Michèle Beau-Faller ${ }^{2,4}$ and Gilbert Massard ${ }^{1}$ \\ ${ }^{1}$ Department of Thoracic Surgery, Strasbourg University Hospital, Strasbourg 67000, France; ${ }^{2}$ Research Unit EA3430, Tumoral \\ Progression and Micro-Environment, Epidemiological and Translational Approaches, Strasbourg University, Strasbourg 67000, \\ France; ${ }^{3}$ Department of Biostatistics, Strasbourg University Hospital, Strasbourg 67000, France and ${ }^{4}$ Department of Molecular \\ Biology, Strasbourg University Hospital, Strasbourg 67000, France
}

Background: We aimed to evaluate whether EGFR mutations (mEGFR) and KRAS amino acid substitutions can predict first site of recurrence or metastasis after non-small-cell lung cancer (NSCLC) surgery.

Methods: Data were reviewed from 481 patients who underwent thoracic surgery for NSCLC between 2007 and 2012.

Results: Patients with KRAS G12C developed significantly more bone metastases compared with the remainder of the cohort (59\% vs $16 \%, P<0.0001)$. This was confirmed in multivariate analysis (MA) (odds ratio (OR): 0.113 ( $95 \%$ confidence interval (Cl): 0.055-0.231), $P<0.0001)$. Significantly, more patients with $m E G F R$ developed liver and brain metastases compared with the remainder of the cohort ( $30 \%$ vs $10 \%, P=0.006 ; 59 \%$ vs $1 \%, P<0.0001$, respectively). These were confirmed in MA (OR: 0.333 (95\% Cl: 0.095-0.998), $P=0.05$; OR: 0.032 (95\% Cl: 0.008-0.135), $P<0.0001$, respectively). Patients with KRAS G12V developed significantly more pleuro-pericardial metastases compared with the remainder of the cohort $(94 \%$ vs $12 \%, P<0.0001)$. This was confirmed in MA (OR: 0.007 (95\% Cl: 0.001-0.031), $P<0.0001$ ). Wild-type patients developed significantly more lung metastases (35\% vs 10\%, $P<0.0001$ ). This was confirmed in MA (OR: 0.383 (95\% Cl: 0.193-0.762), $P=0.006$ ).

Conclusion: Epidermal growth factor receptor mutation and KRAS amino acid substitutions seem to predict site-specific recurrence and metastasis after NSCLC surgery.

Despite many advances in cancer management, lung cancer, with a 5 -year overall survival (OS) rate not exceeding $15 \%$, remains the leading cause of cancer-related deaths worldwide (Bossard et al, 2007). Even in patients who will benefit from surgery, prognosis is not favourable, typically due to the risk of thoracic and extrathoracic recurrence and metastasis (Izar et al, 2014). In many cases, metastases are diagnosed too late, thereby preventing these patients from receiving the benefits of additional surgical therapy. It is now accepted that after thoracic surgery for non-small-cell lung cancer (NSCLC), patients should receive a thoracic computed tomography (CT) scan every 6 months during the first 2 years, and then annually for at least 5 years (Vansteenkiste et al, 2014). However, no recommendations have been made concerning follow-up for brain, bone, liver or adrenal gland metastasis. Indeed, these locations are only typically explored in cases of symptoms, or during the subsequent staging of a thoracic recurrence.

*Correspondence: Dr S Renaud; E-mail: sterenaud0@gmail.com

Received 26 March 2016; revised 27 April 2016; accepted 17 May 2016; published online 23 June 2016

(c) 2016 Cancer Research UK. All rights reserved 0007 - 0920/16 
Consequently, these tumours are frequently diagnosed in multimetastatic patients and are not mitigated by local treatment, such as stereotactic radiation therapy or radiofrequency ablation, which are known to increase OS compared with conventional chemotherapy (Yano et al, 2014).

Recent years have seen an increased understanding of the molecular alterations of cancer cells, and several oncogenic drivers of NSCLC have been identified. These advances have allowed clinicians to adapt medical therapies and achieve, in some cases, significant increases in both disease-free survival (DFS) and OS (Roviello, 2015). Particularly, in metastatic NSCLC, the prognostic and predictive value of epidermal growth factor receptor (EGFR) and $\mathrm{V}$-Ki-ras2 Kirsten rat sarcoma viral oncogene homologue (KRAS) mutations are clearly defined. Indeed, it is now widely known that EGFR mutations (mEGFR) are associated with improved prognosis and prolonged OS and DFS due to the sensitivity of these tumours to EGFR tyrosine kinase inhibitors (Liu et al, 2016). On the contrary, KRAS mutations (mKRAS) are resistant to targeted therapies and are associated with a poor prognosis (Pan et al, 2016). However, it should be noted that the prognostic values of these two mutations on resected NSCLC are not clearly defined (Marks et al, 2008; D'Angelo et al, 2012; Izar et al, 2014; Renaud et al, 2015b). Specifically, the only published meta-analysis of $m E G F R$ contains multiple biases (EGFR mutation rate $>25 \%$ in 14 studies, not reflecting mutation rates in Caucasian populations; 16 studies of Asian patients; and studies of various stages of NSCLC; Zhang et al, 2014b). Nevertheless, previous reports have demonstrated that specific KRAS amino acid substitutions are associated with the activation of different downstream signalling pathways and, consequently, with different clinical behaviours (Ihle et al, 2012). Specifically, depending on the particular KRAS amino acid substitution, cancer cells may respond differently to radiotherapy and/or chemotherapy (Garassino et al, 2011; Janne et al, 2015; Mellema et al, 2015; Renaud et al, 2016) and harbour different prognoses after lung cancer surgery (Renaud et al, 2015b). Furthermore, in colorectal cancer, cancer cells harbouring KRAS mutations are more likely to metastasise to the lung (Renaud et al, 2015c; Shindoh et al, 2016). However, no study has examined the ability of $m E G F R$ and specific KRAS amino acid substitutions to predict site-specific recurrence and metastasis following NSCLC surgery.

We thereby aimed to evaluate whether mEGFR, and more particularly, KRAS amino acid substitutions, were associated with different site-specific recurrence and metastasis patterns after thoracic surgery for NSCLC.

\section{MATERIALS AND METHODS}

The Ethics Committee of the French Society of Thoracic and Cardiovascular Surgeons approved this study (Agreement number: CERC-SFCTCV-2016-2-29-16-57-5-ReSt). The studied population was a part of a cohort published elsewhere and consisted of 841 patients who received NSCLC surgery with curative intent in the Department of Thoracic Surgery at Strasbourg University Hospital (France), from January 2007 to December 2012 (Renaud et al, 2015b). We focused on the 481 patients who experienced thoracic or extra-thoracic recurrence and metastasis after surgery.

Molecular analysis, neo-adjuvant and adjuvant treatment regimens, pre-operative staging and thoracic surgery procedures were performed as previously published (Renaud et al, 2015b).

Covariates and data collection. Baseline patient characteristics were collected, including age, sex, smoking history, and history of neo-adjuvant and adjuvant therapy. The Charlson comorbidity index (CCI), which incorporates 19 chronic diseases weighted according to their association with mortality, was calculated for each patient. We grouped patients into the following established categories according to their total CCI score (Charlson et al, 1987): 0 (no comorbidity); $1-2$ (average); $3-4$ (moderate); and $\geqslant 5$ (severe). Smoking status was characterised as never smoker, $<100$ cigarettes in their lifetime, a former smoker who quit $>1$ year before diagnosis and a current smoker with an ongoing smoking habit or who quit $<1$ year before diagnosis. Wild-type (WT) patients were defined as those harbouring neither mEGFR nor $m K R A S$.

Tumour stage was categorised according to the recommendations of the seventh edition of the American Joint Committee on Cancer Staging Manual. Histopathological characteristics, namely, angio-invasion, R0/R1/R2 and the number of N2 stations involved, were included. Skip metastases were defined as N2 involvement without N1. Microscopic N2 was defined as nodal metastases ranging from 0.2 to $2 \mathrm{~mm}$ in diameter, as previously published (Garelli et al, 2016). Lymph node ratio (LNR) was defined as the ratio of the number of lymph nodes invaded to the total number of resected lymph nodes and categorised according to published guidelines as $\mathrm{LNR}<1 / 3$ and $\mathrm{LNR} \geqslant 1 / 3$ (Renaud et al, 2015a).

Patients were assessed for both local and distant recurrence and metastasis, as well as time to recurrence (TTR). Surgical follow-up consisted of a thoracic CT scan every 6 months during the first 2 years after surgery, and then annually for the remainder of life. In case of recurrence, positron emission tomography scans and brain imaging were performed in order to identify distant metastases. Bone X-rays, brain MRIs or CT scans, abdominal CT scans, and cardiac ultrasonography were performed first only if symptoms were reported. Patients were then categorised according to the site of first recurrence or metastasis: lung, brain, liver, bone, pleuro-pericardial or adrenal gland. In the case of pulmonary or thoracic lymph node recurrence, patients were included in the 'lung group'. In addition, if recurrence or metastasis occurred both in the lung and at other sites simultaneously at the time of diagnosis, the lung was still considered the first site of recurrence, and patients were included in the 'lung group'. Pleuro-pericardial recurrence was defined as pleural and/or pericardial biopsies with histopathological proof of relapse and/or as positive cytology. The date of recurrence or metastases was defined as the first radiographic evidence of cancer relapse upon imaging and/or pathological tumour evidence from a biopsy. The TTR was defined as the time from surgery until the first diagnosis of recurrence or metastasis upon imaging or from biopsy specimens.

Statistical analysis. IBM SPSS (Armonk, NY, USA) v.20 was used for statistical analyses. Comparisons between groups were performed with $\chi^{2}$, Fisher or Student's $t$-tests as appropriate. Cramer's V was used to evaluate correlations between qualitative variables. A multivariate analysis with stepwise logistic regression to predict the first site of recurrence or metastasis was performed for $P$-values $<0.2$ in the univariate analysis. All tests were twosided, and the results were considered significant for $P$ values $<0.05$. The prognostic influence of mutational status on TTR was assessed using the log-rank test.

\section{RESULTS}

Median follow-up time was 39 months (min: 8 months, max: 80 months). Mean age at time of thoracic surgery was 63.39 years $( \pm 11.52)$. Demographic data of the population are presented in Tables 1 and 2. With respect to mutational status, KRAS mutations were observed in 196 patients (41\%). Specifically, we observed 2 G12A mutations (1\%), 91 G12C mutations (46\%), 4 G12D mutations (2\%), 1 G12F mutation (1\%), 3 G12S mutations (2\%), 85 G12V mutations (43\%), 9 G13C mutations (5\%) and 1 
Table 1. Baseline population characteristics

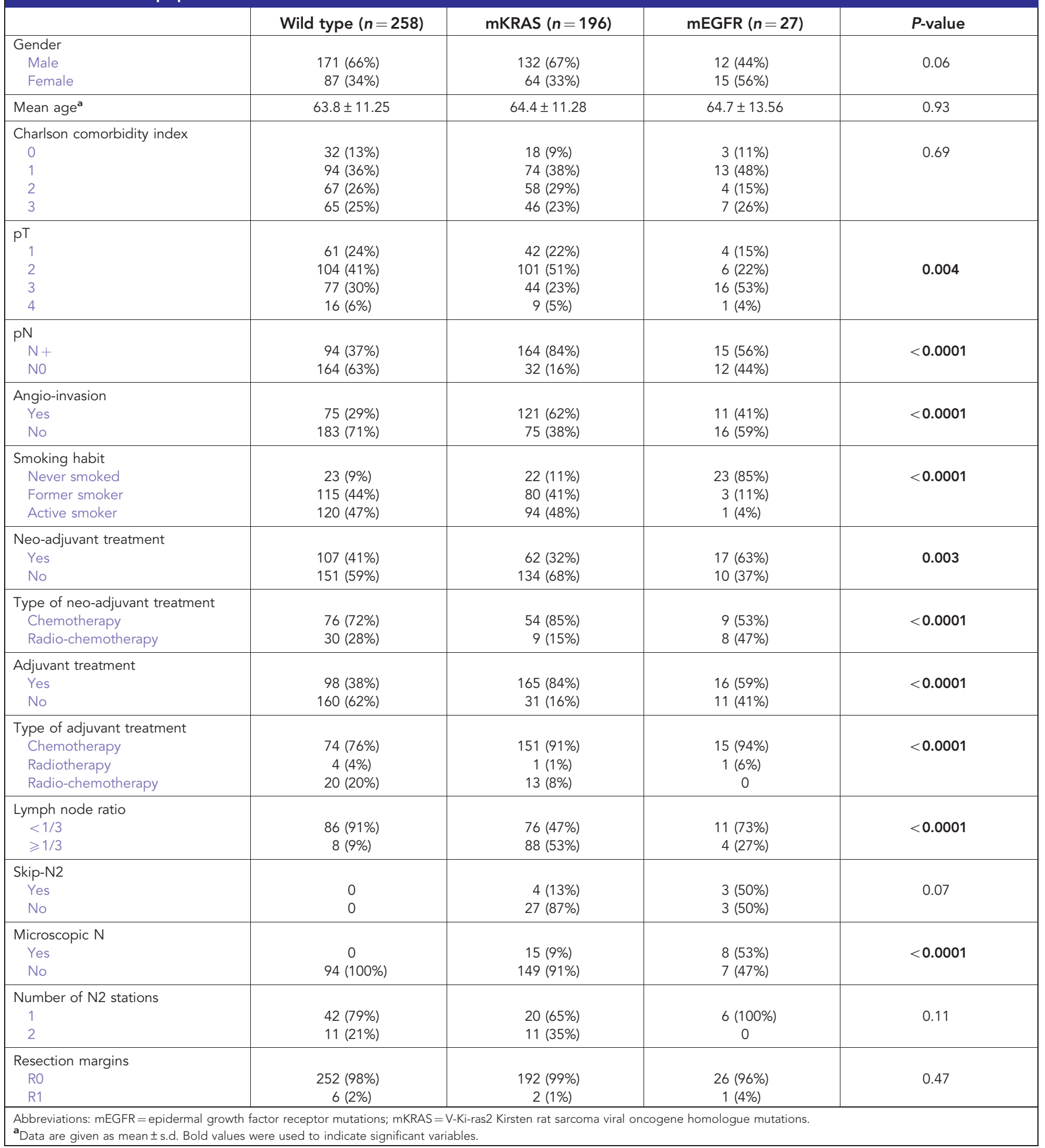

G13D mutation (1\%). In addition, we also observed 27 EGFR mutations (6\%): 1 exon 18 (G791C, c.2155G > T) mutation (4\%), 11 exon 19 deletions (48\%), 4 exon 20 (G796S, c.2386G $>$ A) mutations (17\%), 10 exon 21 (4 L858R) mutations (43\%) and 1 case where exon 21 (L858R) and exon 20 (T790M) were both mutated (4\%).

Recurrence and metastasis. In this cohort, we had previously published that the median TTR was significantly lower for patients with mKRAS compared with WT individuals and mEGFR
$(P=0.01)$, and that type of $m E G F R$ did not significantly influence TTR ( $P=0.97$; Renaud et al, 2015b).

Here we focused on specific KRAS amino acid substitutions. Because KRAS mutations other than G12C and G12V were only observed in few patients, we decided to categorise them into an 'other KRAS mutations' group. Median TTR was significantly different according to the type of KRAS amino acid substitution. Specifically, median TTR was calculated to be 24 months (95\% confidence interval (CI): 21.92-26.07) for patients with G12C mutations, 17 months (95\% CI: 14.09-19.91) for patients with 
Table 2. Baseline population characteristics according to KRAS amino acid substitution

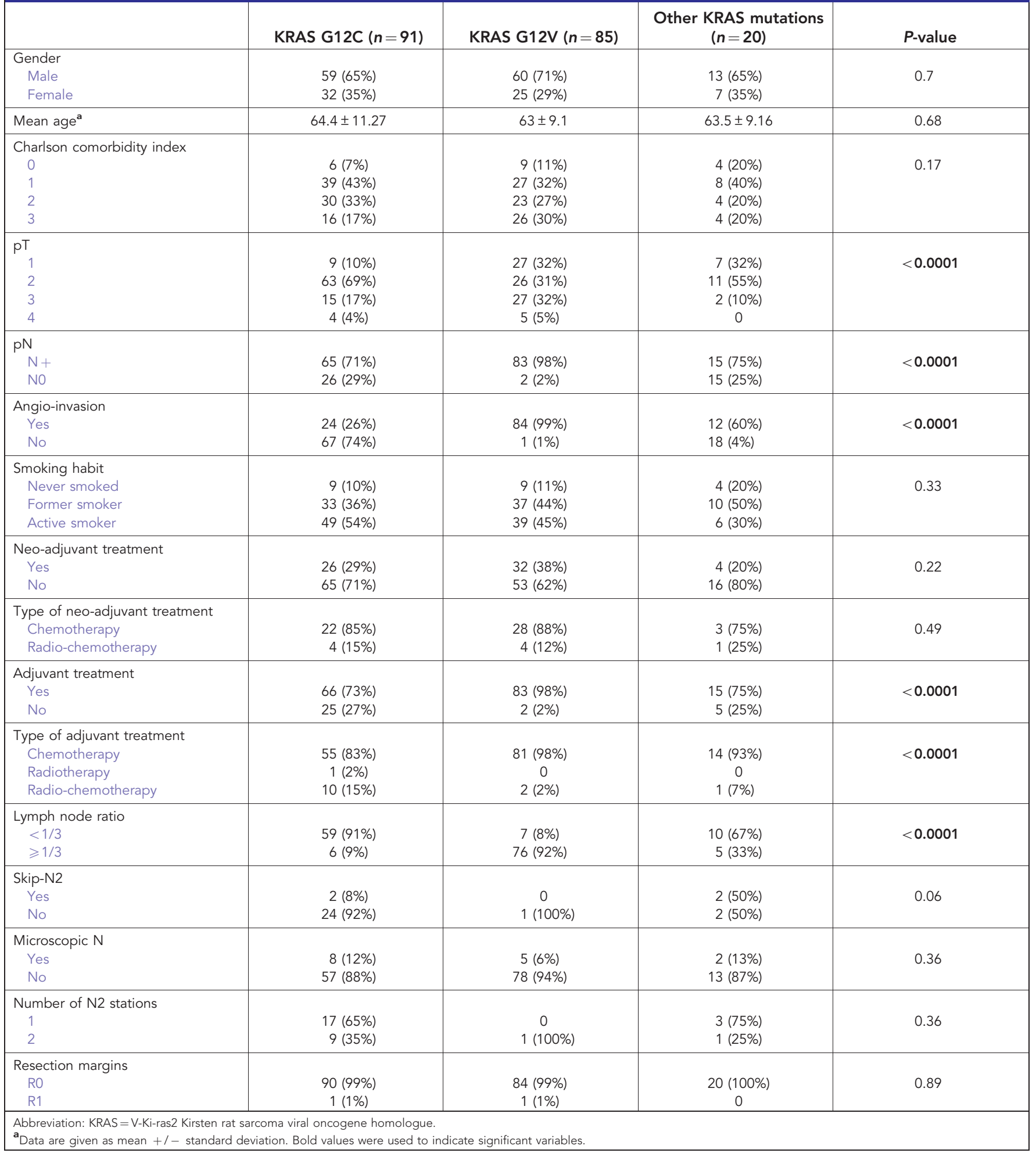

G12V mutations and 15 months (95\% CI: 14.08-15.92) for patients with other KRAS mutations, $P=0.001$ (Figure 1). However, among patients with other KRAS mutations, the median TTR was heterogeneous depending on the specific mutation. Indeed, the median TTR was calculated to be 12 months for patients with G12A mutations, 18 months (95\% CI: 7.22-28.78) for patients with G12D mutations, 14 months for patients with G12F mutations, 32 months for patients with G12S mutations (95\% CI: 7.99-56), 15 months (95\% CI: 6.23-23.76) for patients with G13C mutations and 17 months for patients with G13D patients. Median TTR was not significantly different according to the type of $m E G F R$ $(P=0.97)$.

Site of first recurrence or metastasis. Sites of first recurrence or metastasis were significantly different according to tumour mutational status $(P<0.0001)$. Six patients $(1.3 \%)$ were diagnosed with both lung and other organs metastasis, and were included in the 'lung group'. Data are compiled in Table 3. Data regarding the variables used to predict the recurrence site or metastasis in the univariate analysis are presented in Table 4. 


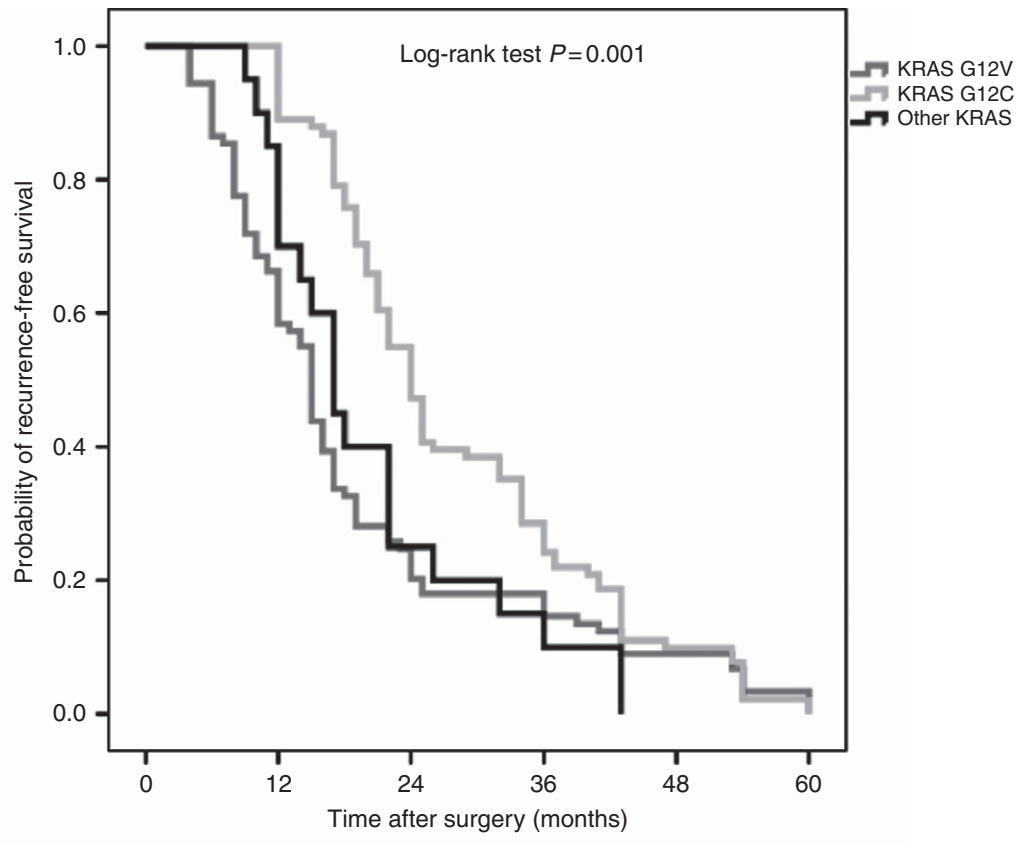

\begin{tabular}{|l|c|c|c|c|c|c|}
\hline KRAS G12V & 85 & 52 & 18 & 13 & 8 & 0 \\
\hline KRAS G12C & 91 & 81 & 43 & 20 & 9 & 0 \\
\hline Other KRAS & 20 & 10 & 5 & 2 & 0 & 0 \\
\hline
\end{tabular}

Figure 1. Kaplan-Meier recurrence-free survival according to KRAS amino acid substitution (i.e., G12C, G12V and other KRAS mutations). A full colour version of this figure is available at the British Journal of Cancer journal online.

\section{Table 3. Site of recurrence according to mutational status}

\begin{tabular}{|l|c|c|c|c|c|c|}
\hline & Bone & Liver & Brain & Pleuro-pericardial & Lung & Adrenal glands \\
\hline Wild type $(n=258)$ & $57(22 \%)$ & $39(15 \%)$ & $26(10 \%)$ & $37(14 \%)$ & $89(35 \%)$ & $10(4 \%)$ \\
\hline EGFR $(n=27)$ & 0 & $8(30 \%)$ & $16(59 \%)$ & $2(7 \%)$ & $1(4 \%)$ & 0 \\
\hline KRAS G12C $(n=91)$ & $54(59 \%)$ & $1(1 \%)$ & $7(8 \%)$ & $9(10 \%)$ & $16(18 \%)$ & $4(4 \%)$ \\
\hline KRAS G12V $(n=85)$ & 0 & $4(5 \%)$ & 0 & $80(94 \%)$ & 0 & $2(1 \%)$ \\
\hline Other KRAS $(n=20)$ & $7(35 \%)$ & $4(20 \%)$ & $2(10 \%)$ & $0(0 \%)$ & $<0.0001$ & 0.13 \\
\hline P-value & $<0.0001$ & $<0.0001$ & $<0.0001$ & $<0.0001$ & \\
\hline $\begin{array}{l}\text { Abbreviations: EGFR }=\text { epidermal growth factor receptor; KRAS }=\text { V-Ki-ras2 Kirsten rat sarcoma viral oncogene homologue. } \\
\text { Data are given as } n(\%) .\end{array}$
\end{tabular}

Bone metastasis. A total of 118 patients (25\%) experienced bone metastasis as the site of first recurrence.

In the univariate analysis, the risk of bone metastasis was higher in patients with G12C mutations compared to all patients in the remainder of the cohort (odds ratio (OR): 7.434 (95\% CI: 4.524-12.217), $P<0.0001)$. Specifically, $59 \%$ of G12C patients experienced a bone metastasis as the first site of recurrence, compared with $16 \%$ of patients in the remainder of the cohort $(P<0.0001)$. In addition, angio-invasion (OR: 0.358 (95\% CI: 0.226-0.569), $P<0.0001$ ), LNI (OR: 0.636 (95\% CI: $0.419-0.965)$, $P=0.04)$ and $L N R \geqslant 1 / 3$ (OR: 0.122 (95\% CI: $0.047-0.319)$ $P<0.0001)$ significantly decreased the risk of bone metastasis. On the other hand, being male $(P=0.96)$, receiving neo-adjuvant treatment $(P=0.18)$ or adjuvant treatment (OR: 0.65 (95\% CI: $0.428-0.987), P=0.055)$ and tumour features such as $\mathrm{R} 0$ resection $(P=0.82)$, skip-N2 $(P=0.45)$ and the absence of microscopic $\mathrm{N}$ $(P=0.71)$ did not significantly increase the risk of bone being the first site of recurrence.
In the multivariate analysis, only non-G12C status (OR: 0.113 (95\% CI: $0.055-0.231$ ),$P<0.0001$ ) and LNR $<1 / 3$ (OR: 3.715 (95\% CI: $1.268-10.882), P=0.02$ ) were independent predictive factors of bone metastasis.

Liver metastasis. A total of 60 patients (12\%) experienced liver metastasis as the site of first recurrence.

In the univariate analysis, the risk of liver metastasis was higher in cases of mEGFR (OR: 3.82 (95\% CI: 1.575-9.265), $P=0.005$ ). Specifically, $30 \%$ of patients with mEGFR experienced liver metastasis as the first site of recurrence, compared with $10 \%$ of patients in the remainder of the cohort $(P=0.006)$. In addition, only angio-invasion (OR: 0.49 (95\% CI: 0.266-0.902)) significantly impacted the risk of liver recurrence. Other variables such as gender $(P=0.81)$, thoracic LNI $(P=0.51)$, neo-adjuvant treatment $(P=0.16)$, adjuvant treatment $(P=0.57)$, LNR $(P=0.09)$, R0 resection $(P=0.64)$, skip-N2 $(P=0.82)$ and 
Table 4. Site of recurrence according to variables included in univariate analysis

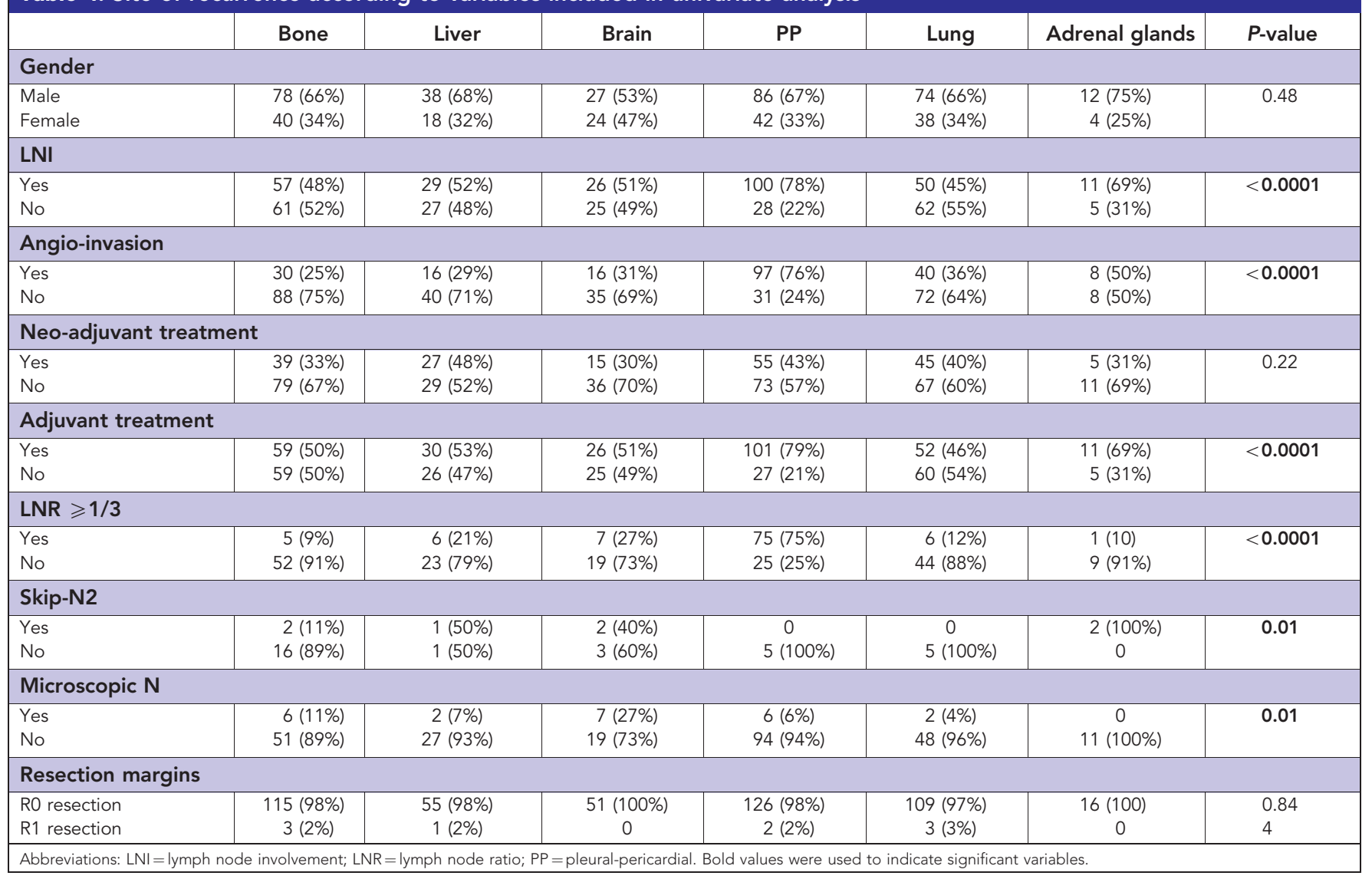

microscopic $\mathrm{N}(P=0.97)$ did not significantly affect the risk of liver recurrence.

In the multivariate analysis, only the absence of mEGFR (OR: 0.333 (95\% CI: $0.095-0.998), P=0.05$ ) could independently predict the liver as the first site of recurrence.

Brain metastasis. A total of 51 patients (11\%) experienced a brain metastasis as the first site of recurrence.

In the univariate analysis, the risk of the brain being the first site of recurrence was significantly higher in patients with $m E G F R$ (OR: 19.017 (95\% CI: 8.03-45.035), $P<0.0001$ ). Specifically, 59\% of patients with $m E G F R$ mutations experienced brain metastasis as the first site of recurrence, compared with $1 \%$ of patients in the remainder of the cohort $(P<0.0001)$. In addition, in the univariate analysis, the risk of brain metastasis was significantly higher in patients with microscopic N (OR: 5.319 (95\% CI: 1.949-14.514), $P=0.001)$. However, gender $(P=0.06)$, thoracic LNI $(P=0.47)$, angio-invasion $(P=0.1)$, R0 resection $(P=0.62)$, neo-adjuvant treatment $(P=0.2)$, adjuvant treatment $(P=0.36)$, LNR $(P=0.39)$ and skip-N2 $(P=0.5)$ did not significantly impact the risk of brain metastasis.

In the multivariate analysis, only the absence of mEGFR (OR: 0.032 (95\% CI: $0.008-0.135), P<0.0001$ ) could independently predict the brain as the first site of recurrence.

Pleuro-pericardial recurrence. A total of 128 patients (27\%) experienced pleuro-pericardial metastasis as the first site of recurrence.

In the univariate analysis, the risk of the pleuro-pericardial region being the first site of recurrence was significantly higher in patients with G12V mutations (OR: 113.306 (95\% CI: 43.745293.482), $P<0.0001)$. Specifically, $94 \%$ of patients with G12V mutations experienced pleuro-pericardial metastasis as the first site of recurrence, compared with only $12 \%$ of patients in the remainder of the cohort $(P<0.0001)$. In addition, in the univariate analysis, the risk of the pleuro-pericardial region being the first site of recurrence was significantly higher in patients experiencing thoracic LNI (OR: 3.568 (95\% CI: 2.245-5.67), $P<0.0001$ ), angioinvasion (OR: 7.048 (95\% CI: 4.437-11.195), $P<0.0001$ ), adjuvant treatment (OR: 3.526 (95\% CI: 2.209-5.630), $P<0.0001$ ) and $\mathrm{LNR} \geqslant 1 / 3 \quad$ (OR: $17.76 \quad$ (95\% CI: 9.553-33.017), $P<0.0001)$. However, gender $(P=0.66)$, neo-adjuvant treatment $(P=0.33)$, skip-N2 (0.59), R0 resection $(P=0.94)$ and microscopic $\mathrm{N}$ $(P=0.39)$ did not significantly impact the risk of pleuropericardial metastasis.

In the multivariate analysis, only non-G12V mutational status (OR: 0.007 (95\% CI: 0.001-0.031), $P<0.0001$ ) was an independent predictive factor of pleuro-pericardial recurrence.

Lung recurrence. A total of 112 patients (23\%) experienced lung metastasis as the first site of recurrence.

In the univariate analysis, the risk of the lung being the first site of recurrence was significantly higher in case of WT status (OR: 4.53 (95\% CI: $2.742-7.484$ ), $P<0.0001$ ). Specifically, 35\% of WT patients experienced lung metastasis as the first site of recurrence, compared with $10 \%$ of patients in the remainder of the cohort $(P<0.0001)$. In addition, there was a negative correlation between G12V status and the risk of lung metastasis (Cramer's V: $-0.242, P<0.0001)$. This result was confirmed in univariate analysis (OR: 0.031 (95\% CI: 0.004-0.222), $P<0.0001$ ), as only $1 \%$ of G12V patients developed lung metastases compared with $28 \%$ of patients in the remainder of the cohort $(P<0.0001)$. Furthermore, the risk of lung recurrence was significantly impacted by thoracic LNI (OR: 0.428 (95\% CI: 0.344-0.809), $P=0.004$ ), adjuvant treatment $(\mathrm{OR}=0.542$ (95\% CI: 0.354-0.83), $P=0.006)$ and LNR (OR: 0.187 (95\% CI: 0.077-0.457), $P<0.0001$ ). However, gender $\quad(P=0.97)$, angio-invasion $(P=0.09)$, $\quad$ R0 resection $(P=0.75)$, neo-adjuvant treatment $(P=0.79)$, skip-N2 $(P=0.59)$ 
and microscopic $\mathrm{N}(P=0.34)$ did not significantly impact the risk of lung recurrence.

In the multivariate analysis, only non-G12V (OR: $17.388(95 \%$ CI: $1.867-161.942), P=0.12$ ) and non-WT status (OR: 0.383 (95\% CI: $0.193-0.762), P=0.006)$ could independently predict lung recurrence.

Adrenal gland metastasis. A total of 16 patients (3\%) experienced adrenal gland metastasis as the first site of recurrence.

Tumour mutational status did not significantly impact the risk of the adrenal glands being the first site of metastases $(P=0.13)$. Specifically, G12V $(P=0.12)$, G12C $(P=0.76)$, EGFR $(P=0.68)$ and WT $(P=0.65)$ status did not significantly impact the risk of adrenal gland metastasis. Only skip-N2 $(P=0.04)$ significantly impacted the risk of adrenal gland metastasis. However, because $100 \%$ of patients exhibiting skip-N2 metastasis also developed adrenal gland metastasis, an OR could not be calculated. Furthermore, gender $(P=0.59)$, thoracic LNI $(P=0.47)$, angioinvasion $(P=0.75)$, neo-adjuvant treatment $(P=0.72)$, adjuvant treatment $(P=0.53)$, LNR $(P=0.11), \mathrm{R} 0$ resection $(P=0.71)$ and microscopic $\mathrm{N}(P=0.64)$ did not significantly impact the risk of adrenal gland metastasis.

In the multivariate analysis, no independent predictive factors of adrenal glands metastasis were identified.

\section{DISCUSSION}

Metastasis and recurrence of NSCLC following lung surgery remains an important cause of death in these patients. It is now accepted that patients presenting with oligometastatic disease experience better prognoses (Ashworth et al, 2014). Indeed, this subgroup of patients can benefit from locoregional treatments, such as surgery or radiofrequency ablation (Yano et al, 2014). However, in the large majority of cases, recurrences and metastasis are diagnosed too late when patients have developed multimetastatic disease and can only benefit from chemotherapy. These outcomes may be attributed to the fact that following thoracic surgery, all patients receive follow-up imaging at the same frequency, only focusing on thoracic field. However, a growing number of publications are highlighting that many tumour types, including NSCLC, are heterogeneous and therefore exhibit different clinical behaviours (Roviello, 2015).

Interestingly, it appears that in $m E G F R$, patients harbouring exon 19 deletions have a better prognosis than individuals harbouring an exon 21 L858R substitution (Zhang et al, 2014a). In addition, it appears that among mKRAS patients, specific amino acid substitutions confer different behaviours to cancer cells. Hence, it seems that some cancer cells are dependent upon KRAS signalling for their survival, whereas others are not (Singh et al, 2009). Moreover, several publications have shown that different KRAS amino acid substitutions are associated with different responses to chemotherapy (Janne et al, 2015; Mellema et al, 2015), radiotherapy (Renaud et al, 2016) or different prognosis in resected NSCLC (Izar et al, 2014; Nadal et al, 2014; Renaud et al, 2015b). In particular, we have previously shown that KRAS G12V mutations were associated with both worse OS and TTR compared with EGFR and WT groups (Renaud et al, 2015b). Furthermore, we have observed that median OS and TTR vary depending on the specific KRAS amino acid substitution present. However, because of the small number of patients harbouring KRAS mutations other than G12C and G12V we were not able to perform a robust statistical analysis. Consequently, it is unsurprising that mutational status could predict site of recurrence and metastasis after NSCLC surgery.
To the best of our knowledge, our study is the first one to highlight that mutational status can predict the first site of disease recurrence and metastasis after NSCLC surgery. Indeed, we have shown that KRAS G12C and G12V transversions are predictive of bone and pleuro-pericardial metastasis, respectively, while the latter is inversely associated with lung recurrence. Furthermore, mEGFR was predictive of both liver and brain metastasis, whereas WT status predicts lung recurrence. These data are in agreement with previous findings (Shindoh et al, 2016), which have shown that after metastasis of colorectal cancer to the liver, rates of further lung metastasis were significantly higher in patients with $m K R A S$. This observation may be attributed to the fact that different downstream signalling pathways might activate different receptors. For example, we have previously hypothesised that over-expression of $\mathrm{C}-\mathrm{X}-\mathrm{C}$ chemokine receptor type $\mathrm{R}$ may underlie the highly aggressive nature of NSCLCs with KRAS G12V transversions, and facilitate the production of chemotactic factors and migration to lymph nodes, vessels and distant organs (Renaud et al, 2015b).

At the end of the 19th century, Stephen Paget developed the 'seed and soil' hypothesis, in which he concluded that some organs may provide a more fertile 'soil' for the development of metastatic 'seeds' (Auerbach, 1988). This theory later evolved into the concept of the tumour microenvironment, which highlights the role of noncancerous stroma and the extracellular matrix in promoting the development of metastases (Wood et al, 2014). Consequently, although more work is needed to more completely understand the ability of specific mutations to predict site-specific recurrence and metastasis, it is reasonable to hypothesise that the activation of different downstream signalling pathways may differentially alter the expression of cellular receptors, thereby 'leading' cancer cells to different organs based on the specific chemoattractants produced in individual tissue microenvironments. However, further in vitro and in vivo studies are required to address these speculations. Nevertheless, our study supports this idea, given that the TTRs of NSCLCs vary according to mutational status and site-specific recurrence and metastasis. Therefore, resected NSCLC specimens should be systematically screened for EGFR and KRAS mutations, and follow-up should be performed according to the specific defect (i.e., a focus on bone metastasis in the event of KRAS G12C mutations; a focus on liver and brain in the event of $m E G F R$; a focus on the pleura and pericardium in the event of KRAS G12V mutations; and a focus on the lungs in patients with WT). Finally, patients with mKRAS should be monitored more closely because of the shorter TTRs observed with the tumours.

It is important to note that our results must be interpreted with caution because of a few limitations of our study. First, this study is a retrospective cohort study of patients receiving treatment from a single medical centre. In addition, the studied population was heterogeneous and included patients with different stages of disease who received varying neo-adjuvant and adjuvant treatment regimens. Furthermore, the 'other KRAS mutations' group was highly heterogeneous and included numerous KRAS amino acid substitutions, all with different median TTRs. However, because of the very small number of KRAS mutations other than $\mathrm{G} 12 \mathrm{C}$ or $\mathrm{G} 12 \mathrm{~V}$, analyses were limited by low statistical power. Similarly, because only a small proportion of patients presented with $m E G F R$, detailed statistical analyses on the different mutations could not be performed. Consequently, we were unable to evaluate the prognostic and predictive values of these mutations.

In conclusion, this is the first study to evaluate the predictive value of individual mutations, particularly KRAS amino acid substitution, on the first site of NSCLC recurrence and metastasis following surgery. Our results indicate that physicians should always screen for EGFR and KRAS mutations, even in the case of resected NSCLCs, due to their prognostic values and ability to influence post-operative follow-up care. These clinical 
modifications may facilitate detection of NSCLC recurrence and metastasis in a timelier manner, thereby affording patients the benefits of locoregional treatment. However, our results must be interpreted with caution because of some limitations of our analysis, and prospective studies are necessary to confirm these preliminary results.

\section{CONFLICT OF INTEREST}

The authors declare no conflict of interest.

\section{REFERENCES}

Ashworth AB, Senan S, Palma DA, Riquet M, Ahn YC, Ricardi U, Congedo MT, Gomez DR, Wright GM, Melloni G, Milano MT, Sole CV, De Pas TM, Carter DL, Warner AJ, Rodrigues GB (2014) An individual patient data metaanalysis of outcomes and prognostic factors after treatment of oligometastatic non-small-cell lung cancer. Clin Lung Cancer 15(5): 346-355.

Auerbach R (1988) Patterns of tumor metastasis: organ selectivity in the spread of cancer cells. Lab Invest 58(4): 361-364.

Bossard N, Velten M, Remontet L, Belot A, Maarouf N, Bouvier AM, Guizard AV, Tretarre B, Launoy G, Colonna M, Danzon A, Molinie F, Troussard X, Bourdon-Raverdy N, Carli PM, Jaffre A, Bessaguet C, Sauleau E, Schvartz C, Arveux P, Maynadie M, Grosclaude P, Esteve J, Faivre J (2007) Survival of cancer patients in France: a population-based study from The Association of the French Cancer Registries (FRANCIM). Eur J Cancer 43(1): 149-160.

Charlson ME, Pompei P, Ales KL, MacKenzie CR (1987) A new method of classifying prognostic comorbidity in longitudinal studies: development and validation. J Chronic Dis 40(5): 373-383.

D’Angelo SP, Janjigian YY, Ahye N, Riely GJ, Chaft JE, Sima CS, Shen R, Zheng J, Dycoco J, Kris MG, Zakowski MF, Ladanyi M, Rusch V, Azzoli CG (2012) Distinct clinical course of EGFR-mutant resected lung cancers: results of testing of 1118 surgical specimens and effects of adjuvant gefitinib and erlotinib. J Thorac Oncol 7(12): 1815-1822.

Garassino MC MM, Rusconi P, Rulli E, Martelli O, Farina G, Scanni A, Broggini M (2011) Different types of K-Ras mutations could affect drug sensitivity and tumor behavior in non-small-cell lung cancer. Ann Oncol 22(1): 235-237.

Garelli E, Renaud S, Falcoz PE, Weingertner N, Olland A, Santelmo N, Massard G (2016) Microscopic N2 disease exhibits a better prognosis in resected non-small-cell lung cancer. Eur J Cardiothorac Surg; e-pub ahead of print 25 February 2016.

Ihle NT, Byers LA, Kim ES, Saintigny P, Lee JJ, Blumenschein GR, Tsao A, Liu S, Larsen JE, Wang J, Diao L, Coombes KR, Chen L, Zhang S, Abdelmelek MF, Tang X, Papadimitrakopoulou V, Minna JD, Lippman SM, Hong WK, Herbst RS, Wistuba II, Heymach JV, Powis G (2012) Effect of KRAS oncogene substitutions on protein behavior: implications for signaling and clinical outcome. J Natl Cancer Inst 104(3): 228-239.

Izar B, Zhou H, Heist RS, Azzoli CG, Muzikansky A, Scribner EE, Bernardo LA, Dias-Santagata D, Iafrate AJ, Lanuti M (2014)

The prognostic impact of KRAS, its codon and amino acid specific mutations, on survival in resected stage I lung adenocarcinoma. J Thorac Oncol 9(9): 1363-1369.

Janne PA, Smith I, McWalter G, Mann H, Dougherty B, Walker J, Orr MC, Hodgson DR, Shaw AT, Pereira JR, Jeannin G, Vansteenkiste J, Barrios CH, Franke FA, Crino L, Smith P (2015) Impact of KRAS codon subtypes from a randomised phase II trial of selumetinib plus docetaxel in KRAS mutant advanced non-small-cell lung cancer. Br J Cancer 113(2): 199-203.

Liu J, Sheng Z, Zhang Y, Li G (2016) The efficacy of epidermal growth factor receptor tyrosine kinase inhibitors for molecularly selected patients with non-small cell lung cancer: a meta-analysis of 30 randomized controlled trials. Target Oncol 11(1): 49-58.

Marks JL, Broderick S, Zhou Q, Chitale D, Li AR, Zakowski MF, Kris MG, Rusch VW, Azzoli CG, Seshan VE, Ladanyi M, Pao W (2008) Prognostic and therapeutic implications of EGFR and KRAS mutations in resected lung adenocarcinoma. J Thorac Oncol 3(2): 111-116.
Mellema WW, Masen-Poos L, Smit EF, Hendriks LE, Aerts JG, Termeer A, Goosens MJ, Smit HJ, van den Heuvel MM, van der Wekken AJ, Herder GJ, Krouwels FH, Stigt JA, van den Borne BE, Haitjema TJ, Staal-Van den Brekel AJ, van Heemst RC, Pouw E, Dingemans AM (2015) Comparison of clinical outcome after first-line platinum-based chemotherapy in different types of KRAS mutated advanced non-smallcell lung cancer. Lung Cancer 90(2): 249-254.

Nadal E, Chen G, Prensner JR, Shiratsuchi H, Sam C, Zhao L, Kalemkerian GP, Brenner D, Lin J, Reddy RM, Chang AC, Capella G, Cardenal F, Beer DG, Ramnath N (2014) KRAS-G12C mutation is associated with poor outcome in surgically resected lung adenocarcinoma. J Thorac Oncol 9(10): 1513-1522.

Pan W, Yang Y, Zhu H, Zhang Y, Zhou R, Sun X (2016) KRAS mutation is a weak, but valid predictor for poor prognosis and treatment outcomes in NSCLC: a meta-analysis of 41 studies. Oncotarget 7(7): 8373-8388.

Renaud S, Falcoz PE, Olland A, Reeb J, Santelmo N, Massard G (2015a) Mediastinal downstaging after induction treatment is not a significant prognostic factor to select patients who would benefit from surgery: the clinical value of the lymph node ratio. Interact Cardiovasc Thorac Surg 20(2): 222-227.

Renaud S, Falcoz PE, Schaeffer M, Guenot D, Romain B, Olland A, Reeb J, Santelmo N, Chenard MP, Legrain M, Voegeli AC, Beau-Faller M, Massard G (2015b) Prognostic value of the KRAS G12V mutation in 841 surgically resected Caucasian lung adenocarcinoma cases. Br J Cancer 113(8): 1206-1215.

Renaud S, Romain B, Falcoz PE, Olland A, Santelmo N, Brigand C, Rohr S, Guenot D, Massard G (2015c) KRAS and BRAF mutations are prognostic biomarkers in patients undergoing lung metastasectomy of colorectal cancer. Br J Cancer 112(4): 720-728.

Renaud S, Schaeffer M, Voegeli AC, Legrain M, Guerin E, Meyer N, Mennecier B, Quoix E, Falcoz PE, Guenot D, Massard G, Noel G, Beau-Faller M (2016) Impact of EGFR mutations and KRAS amino acid substitution on the response to radiotherapy for brain metastasis of nonsmall-cell lung cancer. Future Oncol 12(1): 59-70.

Roviello G (2015) The distinctive nature of adenocarcinoma of the lung. Onco Targets Ther 8: 2399-2406.

Shindoh J, Nishioka Y, Yoshioka R, Sugawara T, Sakamoto Y, Hasegawa K, Hashimoto M, Kokudo N (2016) KRAS mutation status predicts sitespecific recurrence and survival after resection of colorectal liver metastases irrespective of location of the primary lesion. Ann Surg Oncol 23(6): 1890-1896

Singh A, Greninger P, Rhodes D, Koopman L, Violette S, Bardeesy N, Settleman J (2009) A gene expression signature associated with 'K-Ras addiction' reveals regulators of EMT and tumor cell survival. Cancer Cell 15(6): 489-500.

Vansteenkiste J, Crino L, Dooms C, Douillard JY, Faivre-Finn C, Lim E, Rocco G, Senan S, Van Schil P, Veronesi G, Stahel R, Peters S, Felip E (2014) 2nd ESMO Consensus Conference on Lung Cancer: early-stage non-small-cell lung cancer consensus on diagnosis, treatment and follow-up. Ann Oncol 25(8): 1462-1474.

Wood SL, Pernemalm M, Crosbie PA, Whetton AD (2014) The role of the tumor-microenvironment in lung cancer-metastasis and its relationship to potential therapeutic targets. Cancer Treat Rev 40(4): 558-566.

Yano T, Okamoto T, Fukuyama S, Maehara Y (2014) Therapeutic strategy for postoperative recurrence in patients with non-small cell lung cancer. World J Clin Oncol 5(5): 1048-1054.

Zhang Y, Sheng J, Kang S, Fang W, Yan Y, Hu Z, Hong S, Wu X, Qin T, Liang W, Zhang L (2014a) Patients with exon 19 deletion were associated with longer progression-free survival compared to those with L858R mutation after first-line EGFR-TKIs for advanced non-small cell lung cancer: a meta-analysis. PLoS One 9(9): e107161.

Zhang Z, Wang T, Zhang J, Cai X, Pan C, Long Y, Chen J, Zhou C, Yin X (2014b) Prognostic value of epidermal growth factor receptor mutations in resected non-small cell lung cancer: a systematic review with metaanalysis. PLoS One 9(8): e106053.

This work is published under the standard license to publish agreement. After 12 months the work will become freely available and the license terms will switch to a Creative Commons AttributionNonCommercial-Share Alike 4.0 Unported License. 